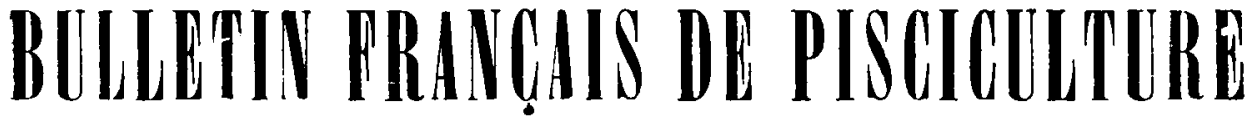

\section{PRISES D'EAU DU XIII ${ }^{\mathrm{e}}$ SIËCLE}

\author{
Par le Comte de Nelfbourg
}

Dix kilomètres de camaux pour alimenter cinquante hectares d'étangs, voilà qui en dit longr sur la misère du moyen àge. Recommencerionsnous? Cela ne " payerait" pas. Or, cela payait. La journée dhomme coûtait denx à trois livres de Carpes ; les viviers ne chòmaient pas ; les gens de tous ćtats achetaient les belles pièces plus qu'ils ne faisaient des moyennes, et la Tanche au double de la carpe. Que les temps sont changés!

Notez que le Ilarengr, l'.lose, le Saumon, le barbeau, l'Inguille faisaient rude concurrence du loisson d'étang. Cependant la cour de Forez, les chevaliers, les magistrats, les riches hombards de Montbrison consommaient, pendant le seul carème, le produit entier d'une centaine d'hectares de bons ctangs appartenant au comte, sans compter la péche dix fois plus considérable des particuliers. I a Carpe marchande était de trois étés et de trois livres. On veillait à n'empoissonner quen " norrain de demipied de longr", bien ćral, que l'on comptait par tìte, à l'unité près. Les pêcheurs avaient des bottes; ie poisson voyageait en tonneaux et parcourait jusqu'à di grandes lieues, bien vif, même au printemps (r). C'était du bon travail. Mais l'histoire de la pisciculture en Forez demande encore des années d'ćtude, et notre présent objet est limilé.

La carle ci-après nous dispense d'explinations détaillées. Trois étangs appartenaient an romte, an matin du mont d't zore, poussée basaltique isolée dans la plaine à une lieve des penles des monts du Forez. Le grand étang a 30 hectares, l Oive $1 \bar{i}$, le petit marais 6 . Le canal, créé à la fin du siècle lernier, n'existant pas alors, la seule eau des pentes pouvait emplir naturellement cé etaugss, ce qui était fort insuffisant pour obtenir une production régulière. On alla donc prendre l'eau du félines, en creusant $2 . \overline{1} 22$ mètres de lossés au long du mont, après avoir établi un barrage à Place Pendue.

Le Félines et le Rangon, son afluent, sont des "gouttes "à débit très irrégulier, d'eau riche coulant des labours et des vignnes, mais souvent peu abondantes.

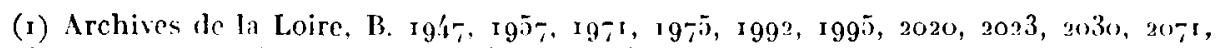
:104, $2105,2171,2182,220 \mathrm{I}$ iे $5,2258,226 \mathrm{r}$ मे $4,2278,2295$, etc. 
On amena donc, en amont de Place Pendue, et de l'autre còté du mont d'Czore, lean du Pralon, ruisseau de meme nature, mais plus important, à se jeter dans le kang̣on, et de là dans le Félines : la prise d'eau des Buissonées est un chef-d'aruve encore admirable dans sa simplicité.

Pour ne rien perdre des ruissellements lluviaux du mont d'Lzore, on récolta l'eau des pentes opposées, el celle de l'étang de la Vernay, au lieu de la laisser s'écouler vers le Lignon, an nord. Ce travail compliqué est le seul de tout le système qui ne subsiste plus, le ranal molerne l'ayant rendu inutile; mais l'eau du canal. cest-ì-dire de la looire, ne vaut pas celle de la Vernay.

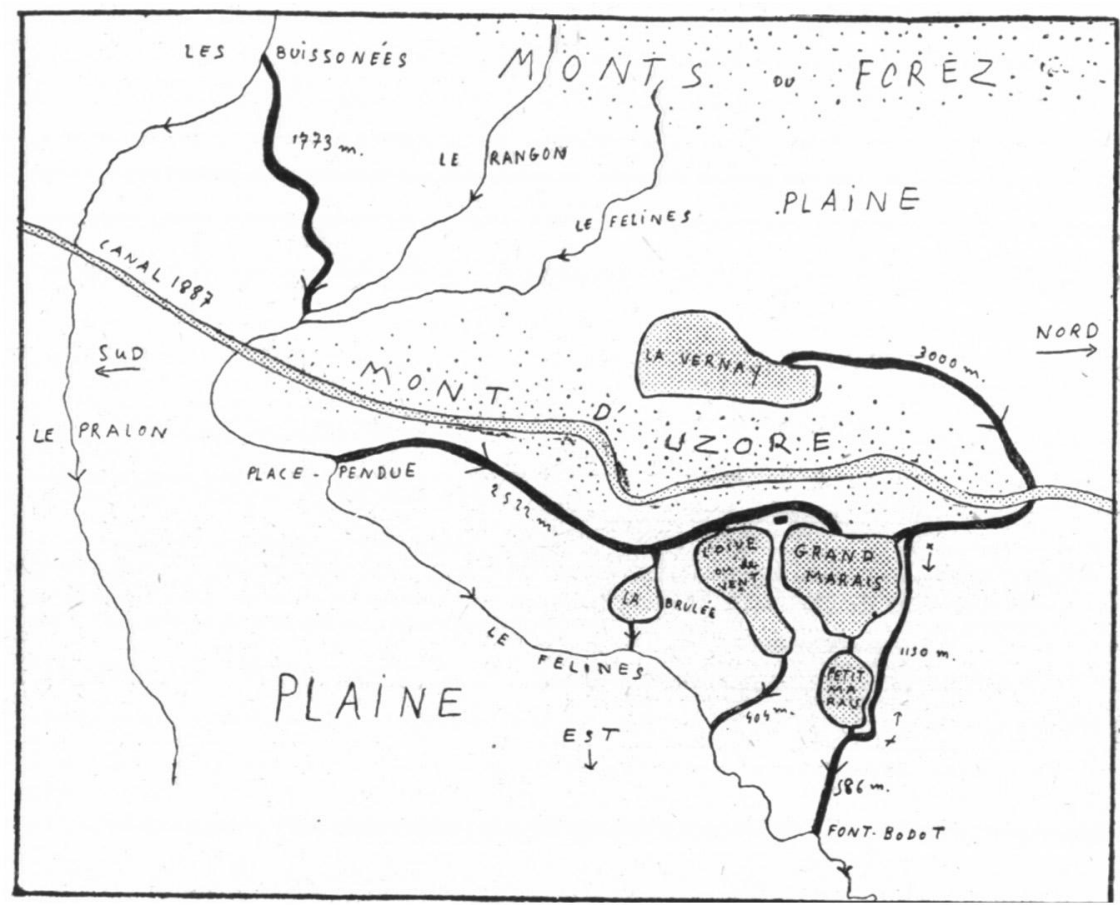

Figure 2. - Les élangs d'Uzore

Voilà donc les étangs remplis par trois dérivations savantes : d'autant plus savantes que Place Pendue est à 370 mètres d'altitude, et la prise d'eau du grand marais à $36 \mathrm{r}$ mètres, soit du o,36\%. Les Buissonées sont à 395 , la Vernay à 400 .

Les étangs du comte, depuis étangs du roi, comme Vidrieu, n'ont pas de chaussées : vider cette cuvette est impossible sans creuser une pente. Le petit marais fut donc ajusté ; son plein est à un pied sous le niveau plein du grand marais. De la bonde du petit marais, il fallut gagner un point du Félines permettant un "tirage ". On piqua presque droit, par un fossé qui mesure de 4 à 5 mètres de profondeur. Celui qui vide l'Oive est moitié moins encaissé.

Il faut noter que ces étangs se vidaient normalement, puisque les comptes du xrv $^{\mathrm{e}}$ siècle portent peu de frais de curage. Ils avaient des 
bondes à "poltron ", le vieux bouchon de bois, au plus bas de l’étang̨, et la pression d'eau du début de lit làche dégagreail le fossé. Fn 17,2 , on établit de belles rainures en pierre de taille, et depuis, avec le sıstème des plateaux superposés, la vase reste dans les fossés et dins l'étang, occasionnant de très grands frais de curage cxlerne et interne. I.e mosen ìge avait du bon...

Comme on pèchait jadis ces étangs en phusicurs fois, en filetant le fossé intérieur, on craignit les "coups d'eau ", le ruissellement des pentes nord, et l'on garantit le grand et le pelit marais par un fossé de garde qui les borde du côté du danģer.

Ces trois élanģs, alimentés en eaux riches, cultivés parfois (on y semait du froment trémois), foumissaient, au $x v^{-0}$ siècle, une moyenne assez récrulière de 250 quintaux de poisson, dont $2 / 5$ de Tanches, ce qui est extraordinaire, et demeura fort avantageux jusque vers la guerre. Réputés excellents, ils produisaient, sous la sage administration de M. DE LA Plagie, aux environs de $\_880$, au moins 108 quintaux de Carpes et $; 8$ de Tanches, au plus 221 de Carpes et 2ho de Tanches. Hélas! le canal apporta sa trompeuse richesse d'eau assurée. Pourquoi courir aux Buissonées? P'ourquoi lutter pour conserver l'cau de la lernay? L'eau de Loire remplaça trop souvent celle des goultes grasses. On tomba jusquà 42 quintaux de Carpes et 34 de Tanches. Enfin, I'an dernier, un citadin chasseur ohtint un total de 40 quintaux. Barbare moyen âge !

Le propriétaire actuel remontera la pente : mais il est prouvé qu'il n’est pas de bon étang sans bon Iisciculteur.

Nous n'avons pas la date de création des étangrs du comte à lzore, mais leur aménagement rappelle celui de Vidrieu, et l'on suppose que ces 1ravaux datent environ de l'an 1250.

Un des titres les plus anciens était demeuré caché, et nous l’avons récemment découvert. En voici la traduction du latin :

(Original parchemin, jadis scellé, Archives nationales, 1'. 49. 8 ociobre 1317)

Nous, garde du scel royal en la seneschausse de Lyon, savoir faisons à fous qui ces présentes lettres verront : comme Jean d'Ysore réclamait d illustre homme messire Jean, comte de Forez, que, ayant naguère pêché et fait pêcher les inangre dudit comte a $\chi$ sore et ailleurs toules ies fois que ce fut utile, il ne lui en fut fait nul payement.

Cependant ledit seigneur comte et Johannin d'Ysore, constilués devant notre notairejuré, Symon Oudin de Beaulieu, clerc du roi, firent mtre eux, spontanément, les pacte et convention suivants : Ledit seigneur conte sera tenu de payer audit Johannin incontinent 18 livres viennois en rícompense de ses labeurs passés, laquelle somme ledit Joannin confesse avoir resue dudit comte et en quitte ledit conte et les siens à jamais.

Ii est encore convenu que ledit Johannin doit pêcher, par soi ou par autrui, comme et quand il faudra, à ses propres frais et arec ses propres filets qu'il devra faire fabriquer à son compte, tous les ćtangs que le comte a soit à Ysore, soil ailleurs en sa comté de Forez.

Et pour cela ledit seigneur comte sera tenu de domner andit Johannin, chaque annóc en la Saint Martin d'hiver, 6 livres viennois of um robe de pinne, de celle dont il vet ics valets de sa chambre. Ledit comte devrit aussi pourvoir de vivres ledit Johannin et ses domestiques à lui nécessares pour pêcher les étangs du cum!e en Forez, excepté les 
étangs d'Ysore que ledit Johannin doit pêcher et y faire le nécessaire et procurer les vivres et tout ce que besoin sera à ses frais, comme il est convenu.

Il est encore convenu que la pièce de terre existant entre les deux étangs d'Ysore, terre que ledit Johannin d'Ysore tient de la directe seigneurie et censive du comte, retournera, libre, au comte et aux siens à perpétuité, mais que s'il advient que ie comte ou les siens abénévisent ladite terre, alors ledit Johannin d'Ysore ou ses héritiers pourront avoir ladite terre avant tous autres, sous le même cens qu'un autre offrira et sous les mêmes conditions qu'elle serait cédée à un autre.

Promettant ledit comte et ledit Johannin par mutuelies stipulations, devant ledit notaire, de bonne foi et sous l'obligation de tous leurs biens, d'observer tout ce que dessus à jamais et inviolablement et de n'y contrevenir en rien. Fienonçant ledit comte et ledit Johannin de ce fait et de bonne foi a toute action et deception de dol et de tout droit canon et civil et au droit disant que générale renonciation ne vaut.

En témoin de quoi, à ia demande desdits comte et Johannin, à nous rapportée par ledit notaire juré en lequel sur ce nous avons pleine fiance, nous avons fait apposer le scel royal en ladite sćnéchaussée de Lyon aux présentes.

Donné le $8^{\circ}$ jour d'octobre, l'an du seigneur 1317 , en présence de Simon Breton et de Guillaume Faure, clercs. Seing dudit juré : Symon.

La terre que Jeannin rend au comte, et qui appartient encore aux étangs, mesure II hectares, donne goo bichets de froment, soit pour Jeannin un bénéfice d'environ 500 bichets, une moyenne de 25 livres tournois et Io livres en comptant avec l'assolement de l'époque. Sa situation ne semble donc pas changée par l'accord.

Pour aider à se faire une idée, notons, qu'en 1399 , la toise de curage valait 3 deniers ou $x / 4$ de sol, et 4 sous en rgoo, 40 sous en 1929 ; la Carpe 2 sous le kilo, en 1399 et 120 en I $^{2} 9$; la journée de pêcheur 2 sous $\mathrm{r} / 2$ en $\mathrm{r} 399$ et 500 sous en r929. Les prix s'étaient élevés de 13 r 7 à ı 399, mais en I 446, „ux mèmes lieux, nous trouvons encore la Géline à I sol, le Conil à 20 deniers, le Lièvre à 2 sols, le hichet de froment à moins de 2 sols : ce qui donne une haute idée du prix de la Carpe au temps de Joannin d'Uzore!

Les étangs du comte de Forez furent confisqués au profit du roi en ${ }_{1} \bar{j}_{2} 6$, lorsque le Parlement, par un déni de justice, réunit le Forez à la couronne. Ils prirent, au $\mathbf{x v I I}^{\circ}$ siècle, le nom, qui leur reste, d'étangs du roi.

En I648, la Carpe marchande, de I à 2 livres apparemment, vaut 25 livres le cent, environ 6 sous le kilo.

Fn 1687 , réparés à neuf, les trois étanģs sont loués 850 livres, et 700 livres en 1713 . Leur rendement devait donc être tombé à moins de 200 quintaux.

En I 749 , le fermier revendique encore la prise d'eau de la Vernay. Peu après, les étangs du roi furent vendus à M. DE Cuatcayt, conseiller au bailliage, qui possédait un domaine voisin. On grava dans la pierre du barrage des Buissonets cette inscription, qui subsiste :

"Prise d'eau des étangrs du roi vendus en 1765 par le roi Louis XV à Monsieur Challaie. Mise en possession en $\mathbf{r}_{72}$ par M. de Meaux, lieutenant général du bailliage et jugre domanial du comté de Forez, et Mon. sicur de la Chaize, procureur du roi ". 
Chalbaye eut, entre autres enfants, Me Raver, baronne de Mloxtagre,

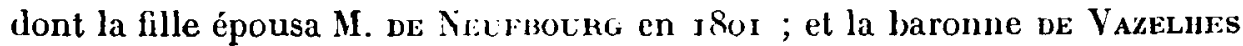
dont les enfants vendirent les étangs du roi à M. de LA PI.Agne, fameux pisciculteur qui conservait au $\mathbf{x i x}^{\mathrm{e}}$ siècle les traditions excellentes du $\mathbf{x i r}^{\ominus}$.

Ce sont elles qu'il s'agit de faire revivre en 1930 .

\title{
LES FOURS A COKE ET LA POLLUTION DES RIVIÈRES
}

\author{
Par M. Etienne HUbaUlT
}

Inspecteur des Laux et Forèts,

Assistant de Zoologie à la Station de Recherches de l'Ecole nationale des Eaux et Forêts.

Duns la région de l'Est, pays de grande industrie, sillonné de rivières importantes, le plus souvent riches encore en Poisson, it n'y a pour ainsi dire pas d'année qu'il ne se produise d'empoisonnements massifs du fait des déversements des usines. Certaines partics de cours d'eau commencent même à être complètement dépeuplées.

A la demande du Service des Eaux et Forêts et à la requête des Tribunaux, j'ai été appelé à m'occuper de cette question, en collaboration avec d'autres personnes. Maintenánt que les comptes rendus administratifs et que les rapports d'expertise ont été déposés et distribués, qu'ils sont pour ainsi dire tombés dans le domaine public, je désirerais attirer l'atlention sur des eaux résiduaires qui m'ont paru particulièrement nocives : celles des fours ì coke, estimant peul-èlre moins dangereuses celles que rejettent les hauts fourneaux, souvent proches voisins des cokeries (1).

C'est au cours de l'automne de 1927 que l'attention du Service local des Eaux et Forêts de Nancy fut attirée sur le dépeuplement complet du cours inférieur d'un petit affuent de la Moselle, du fait du déversement des usines d'une importante firme métallurgique, affluent dont elles se servent comme d'égout collecteur. L'usine principalement visée était une cokerie nouvelle qui commença à fonctionner au début de $19^{25}$.

Ceci m'amena à faire suir place une première étude ; je n'y reviendrai que brièvement, puisqu'elle fut déjà l'objet d'une publication (2).

(1) Les eaux résiduaires des liauts fourneaux ont fait l'objet d'un travail de I'. Grf́lot. La pollution des rivières par ies eaux résiduaires des hauts fourneaux. Bull. des Sc. pharmacol., XXXL, p. 520, 19\%'. - Jo ne saurais mettre en doute les sombrcases analyses de l'auteur. Néanmoins, all cours de scs expériences sur des l'oissons, il m'a paru s'êtro placé dans des conditions un peu artificielles.

(y) Derow (C.) et Hubault (lir.) - Les déversements des eaux résiduaires des fours à coke dans les rivières de l'Est, el la faune piscicole. - C. R. Acad. d'Agricult., XIV, p. $449,: 928$. 